\title{
DETERMINATION OF ANTIBIOTIC RESIDUES IN MILK BY MICROBIAL INHIBITORY TESTS
}

\author{
Juščáková, D., Kožárová, I. \\ University of Veterinary Medicine and Pharmacy, Komenského 73, 04181 Košice \\ Slovakia \\ ivona.kozarova@uvlf.sk
}

\section{ABSTRACT}

Undesirable substances enter the organism of animals mostly via feed, water or veterinary medicines and their residues pass subsequently into the products of animal origin. In dairy cows, sheep and goats these residues are eliminated particularly in milk. Milk intended for human consumption must comply with safety criteria also with respect to residues of antibiotics. The aim of this study was to determine the presence or absence of antibiotic residues in the milk using the tests Milchtest and Premi ${ }^{\oplus}$ Test. While the Milchtest was developed for the determination of antibiotic residues in cow, sheep and goat milk, the Premi ${ }^{\oplus}$ Test is intended for the determination of antibiotic residues in meat juice, liver, kidneys, fish, eggs and in the urine of animals treated with antibiotics. As examined matrices, we used 45 samples of raw cow's milk collected at 3 agricultural farms and 10 samples of milk offered to consumers at grocery stores. When using the Milchtest, 8 samples tested positive and 10 provided dubious results while testing with the Premi ${ }^{\mathrm{e}}$ Test showed that only 6 samples were posi- tive for antibiotics. Comparison of the results confirmed a higher detection sensitivity of Milchtest reflected in higher numbers of positive samples and the detection of dubious results in samples of raw cow's milk. However, it should be noted that even the Premi ${ }^{\circledR}$ Test, although not intended preferably for the determination of antibiotics in milk, can be used, if needed, for the preliminary screening of antibiotic residues in such a matrix.

Key words: antibiotics; milk; residues; screening

\section{INTRODUCTION}

Milk is considered one of the most valuable foods and plays an important role in the life of animals and especially in humans. It is the first source of nutrition for infant mammals before they are able to digest other types of food. Therefore it is important to ensure the highest possible nutritional and hygiene standard of milk [5].

Milk is a valuable source of important substances such as milk fat, proteins, lactose (milk sugar), vitamins and 
minerals. These substances are unique for the human body providing a considerable proportion of energy and nutrients. Milk fat is easily absorbable and digestible. Milk proteins are a source of a number of essential amino acids essential for the organisms. Lactose is a valuable and easily digestible source of energy, which also supports the regulation of gut function.

According to the Regulation (EC) No. 853/2004 of the European Parliament and the Council [8], laying down specific hygiene rules for food of animal origin, raw milk must originate from animals that show no signs of infectious diseases transmissible to humans via milk. The animals must be in good health, show no clinical signs of diseases that may contaminate milk, they should not suffer from detectable udder inflammation, nor udder trauma that can affect the milk. The animals should not be administered any unpermitted substances or medications and if they were administered permitted substances or medications, the withdrawal periods prescribed for these substances or medicines must be observed [8].

The use of antibiotics for the treatment of animals kept for the production of food of animal origin can result in the presence of residues of these substances in the products, including the milk, pass through the food chain directly to the consumer and seriously affect human health. In the interest of protecting consumer's health and ensuring food safety, European legislation specifies a maximum residue limits (MRL) for all pharmacologically effective substances administered to animals kept for the production of food of animal origin. MRL is defined as the maximum concentration of residue of pharmacologically active substance accepted in a food product as a guarantee of its safety $[1,9]$.

Antibiotics belong among pharmacologically active substances with potent health risks. As their finding in food of animal origin is not rare, the development of reliable screening methods that can detect their presence in food appears essential. Currently, there are available many screening methods differing in: the type of the test strain used; susceptibility of test strains to individual types or groups of antibiotics; and the determination procedures and temporal or financial demands [7].

Milchtest (Packhaus Rockmann, Germany) is one of the most recent microbial inhibitory tests developed for the determination of antibiotic residues in raw, thermally treated and dried cow, sheep and goat milk. It is simple, provides accurate results and exhibits adequate sensitivity in the determination of residues of: beta-lactams, tetracyclines, macrolides, aminoglycosides, sulphonamides and trimetoprim at the level of MRL. Version Milchtest MT 25 FARM is recommended for small milk producers for the purpose of self-control and Milchtest MT 50 was developed for large producers and industrial milk-processing units. Flexible microplates MT 96 FP and MT 288 FP are recommended for the examination of a greater number of milk samples and official controls. Just as the Premi ${ }^{\oplus}$ Test, the Milchtest also combines the principle of agar diffusion tests with indicator colour changes due to the active metabolism of the test strain Bacillus stearothremophillus var. calidolactis at inhibitor absence. The blue-violet colour of the agar nutrient medium indicates the presence of inhibitory substances (antibiotics) in the tested sample [3].

The Premi ${ }^{\oplus}$ Test (R-Biopharm AG, Germany) is a broadspectrum microbiological screening test intended for the determination of antibiotic residues in food and raw materials of animal origin. It is usable also for the determination of antibiotic residues in feed and body fluids (urine). The Premi ${ }^{\oplus}$ test allows one to determine the presence of the most important groups of antibiotics ( $\beta$-lactams, cephalosporins, macrolides, tetracyclines, aminoglycosides) at or below their MRL. Premi ${ }^{\circledR}$ Test also combines the principle of agar diffusion tests with changes in an indicator colour due to the active metabolism of the test strain in the absence of an inhibitor. The tested sample is dosed to vials containing the test strain Bacillus stearothremophillus var. calidolactis. Normal growth of the test strain during incubation results in a colour change of a $\mathrm{pH}$ indicator from blue-violet to yellow. If the sample contains substances inhibiting the growth of the test strain (antibiotics), the blue-violet colour of the indicator remains unchanged. The Premi ${ }^{\circledR}$ Test is available in two versions, Premi ${ }^{\circledR}$ Test 25 and Premi ${ }^{\oplus}$ Test $4 \times 25[4,10]$.

With regard to the fact that both tests are based on the inhibition of growth of the test strain Bacillus stearothremophillus var. calidolactis and are performed using the same principle, conditions of application and incubation of samples, and evaluation of results with the exception of the tested food matrices, we decided to use these two microbial inhibitory tests for determination of the presence or absence of antibiotics in milk intended for human consumption and compare their performances. 


\section{MATERIALS AND METHODS}

During 2016 and 2017, we tested 45 samples of raw cow's milk collected at 3 agricultural farms registered in Slovakia and 10 samples of milk produced in Slovakia and Czechia and offered to consumers at grocery stores. The samples were stored in a freezer at $1-8^{\circ} \mathrm{C}$. Immediately before analysis, the samples were defrosted and mixed thoroughly. They were examined for the presence of residues using the procedures specified by the manufacturers of Premi ${ }^{\oplus}$ test and Milchtest.

Milchtest: Using disposable tips, $50 \mu \mathrm{l}$ aliquots of the samples were transferred to vials supplied with the test kit. The vials were marked, sealed with foils supplied by the manufacturer as a part of the test kit and transferred to a thermoblock (Dry Bath EMK 20, Euroclone S.p. A, Pero, Italy) where they were incubated for about 3 hours at a temperature of $65 \pm 0.5^{\circ} \mathrm{C}$. The test was terminated when the colouring of the agar medium of the negative control changed from violet to yellow. The results were evaluated using a colour scale supplied by the manufacturer. Yellow colour indicated the absence of antibiotics in the sample, while violet colour indicated the presence of antibiotic residues. Yellow-green or yellow-violet colouring of the agar medium indicated the presence of antibiotics at a level equal to the test detectability level.

Premi ${ }^{\circledast}$ Test: By means of a micropipette with a defined volume, $100 \mu \mathrm{l}$ aliquots of a milk sample were transferred into the provided vials. The vials were marked, sealed with foils supplied by the manufacturer and transferred to a thermoblock (Acublock Digital Dry Bath D 1200, Labnet, Edison, USA) where they were incubated for approximately 3.5 hours at a temperature of $64 \pm 0.5^{\circ} \mathrm{C}$. The test was terminated when the colouring of the agar medium of the negative control changed from violet to yellow. After incubation, we examined the vials for a positive or negative reaction. A yellow colour indicated the absence of antibiotics in the sample, while a violet colour indicated the presence of antibiotic residues. Yellow-violet colouring of the agar medium indicated the presence of antibiotics at a level equal to the test detectability level.

\section{RESULTS AND DISCUSSION}

Samples of raw cow's milk and milk obtained from grocery stores were tested for the presence of antibiotic resi- dues. Also, the suitability of both methods for the rapid screening of antibiotic residues in milk were compared.

When using the Milchtest, a positive result was obtained in 8 samples of milk, while using the Premi ${ }^{\oplus}$ Test only 6 samples were positive. The positive reaction indicating the presence of residues exceeding the level of test detectability was manifested by the violet colour of the agar medium, as the colour of the $\mathrm{Ph}$ indicator remained unchanged due to the inhibition of growth of the test strain (Bacillus stearothermophilus var. calidolactis) by the presence of the antibiotics.

Negative results were indicated by the yellow colour of the agar medium resulting from the metabolic activity of the test strain and relevant change in the indicator colouring from blue-violet to yellow. When using the Milchtest, 37 samples were negative, while the Premi ${ }^{\circledR}$ Test indicated negativity in 49 of the samples.

Other colour shades from yellow, yellow-green to yellow-violet, which did not allow us to decide unambiguously whether the samples were positive or negative, resulted from the inhibition of the growth of the test strain by the presence of antibiotic at the level of test detectability or immediately below it. Such samples were considered dubious and must be subjected to confirmation analysis for confirmation or negation of the positive result. When using the Milchtest, we obtained 10 dubious results, while there were no dubious results with the Premi ${ }^{\circledR}$ Test.

The results obtained in this study are presented in Tables $1-4$ and Figures $1-8$.

The basic aim of food legislation is to prevent any health risks associated with food. The Regulation (EC) No. 853/2004 sets safety criteria for raw milk. These criteria involve the so-called trigger values - if any of them is exceeded, the operators of the food processing establishments are obliged to take corrective measures and report this to the relevant authority. Animals treated with antibiotics that could result in excretion of their residues in milk must be identified so that the milk produced by them before the end of withdrawal period is not used for human consumption. This means that raw milk containing residues of antibiotics in amounts exceeding MRL permitted according to Commission Regulation (EU) No. 37/2010 or milk containing combination of residues exceeding in total any maximum acceptable value must not be placed on the market $[1,8]$.

In our study we used two microbial inhibitory tests to determine presence/absence of antibiotics in raw cow's 
Table 1. Results of the examination of raw cow's milk for the presence/absence of antibiotic residues using the Milchtest and Premi ${ }^{\oplus}$ Test - Farm 1

\begin{tabular}{|c|c|c|c|c|c|c|c|c|c|c|c|c|c|c|c|}
\hline \multirow{2}{*}{ Test } & \multicolumn{15}{|c|}{ Sample } \\
\hline & 1 & 2 & 3 & 4 & 5 & 6 & 7 & 8 & 9 & 10 & 11 & 12 & 13 & 14 & 15 \\
\hline Milchtest & + & + & - & + & \pm & - & - & - & - & - & + & - & - & - & - \\
\hline Premi ${ }^{\circledR}$ Test & - & - & - & - & - & - & - & - & - & - & + & - & - & - & - \\
\hline
\end{tabular}

+ - positive; \pm - dubious; - - negative

Table 2. Results of the examination of raw cow's milk for the presence/absence of antibiotic residues using the Milchtest and Premi ${ }^{\oplus}$ Test - Farm 2

\begin{tabular}{|c|c|c|c|c|c|c|c|c|c|c|c|c|c|c|c|}
\hline \multirow{2}{*}{ Test } & \multicolumn{15}{|c|}{ Sample } \\
\hline & 1 & 2 & 3 & 4 & 5 & 6 & 7 & 8 & 9 & 10 & 11 & 12 & 13 & 14 & 15 \\
\hline Milchtest & \pm & - & \pm & - & - & - & - & \pm & - & + & - & + & - & - & \pm \\
\hline Premi ${ }^{\oplus}$ Test & - & - & - & - & - & - & - & - & - & + & - & + & - & - & - \\
\hline \multicolumn{16}{|c|}{+- positive; \pm - dubious; -- negative } \\
\hline \multicolumn{16}{|c|}{$\begin{array}{l}\text { Table 3. Results of the examination of raw cow's milk for the presence/absence } \\
\text { of antibiotic residues using the Milchtest and Premi }{ }^{\circledR} \text { Test - Farm } 3\end{array}$} \\
\hline & \multicolumn{15}{|c|}{ Sample } \\
\hline & 1 & 2 & 3 & 4 & 5 & 6 & 7 & 8 & 9 & 10 & 11 & 12 & 13 & 14 & 15 \\
\hline Milchtest & \pm & - & - & - & - & \pm & - & - & - & \pm & + & + & - & + & \pm \\
\hline Premi ${ }^{\oplus}$ Test & - & - & - & - & - & - & - & - & - & + & - & + & - & + & - \\
\hline
\end{tabular}

+ - positive; \pm - dubious; - - negative

Table 4. Results of the examination of cow's milk for the presence/absence of antibiotic residues using the Milchtest and Premi ${ }^{\oplus}$ Test - grocery store

\begin{tabular}{cccccccccccc}
\hline & \multicolumn{10}{c}{ Sample } \\
\cline { 2 - 10 } Test & 1 & 2 & 3 & 4 & 5 & 6 & 7 & 8 & 9 & 10 \\
\hline Milchtest & - & - & - & - & - & - & - & - & - & - \\
Premi®Test & - & - & - & - & - & - & - & - & - & - \\
\hline
\end{tabular}

$$
-- \text { negative }
$$




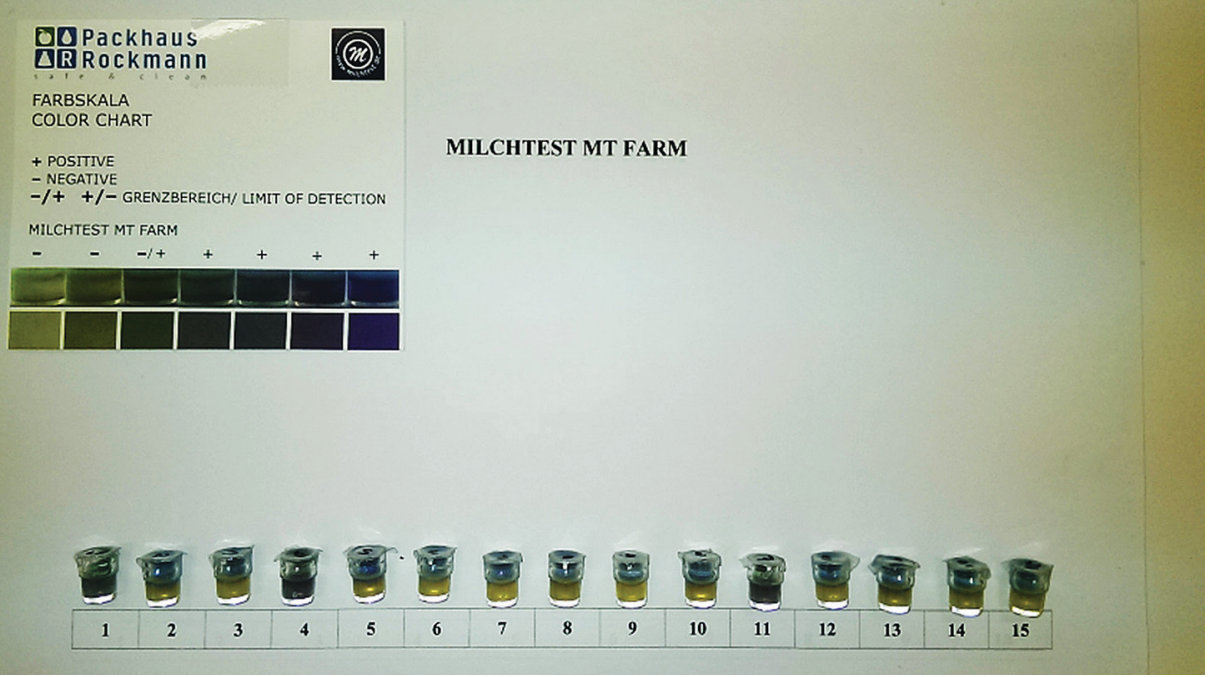

Fig. 1. Examination of raw cow's milk for the presence/absence of antibiotic residues using the Milchtest - Farm 1

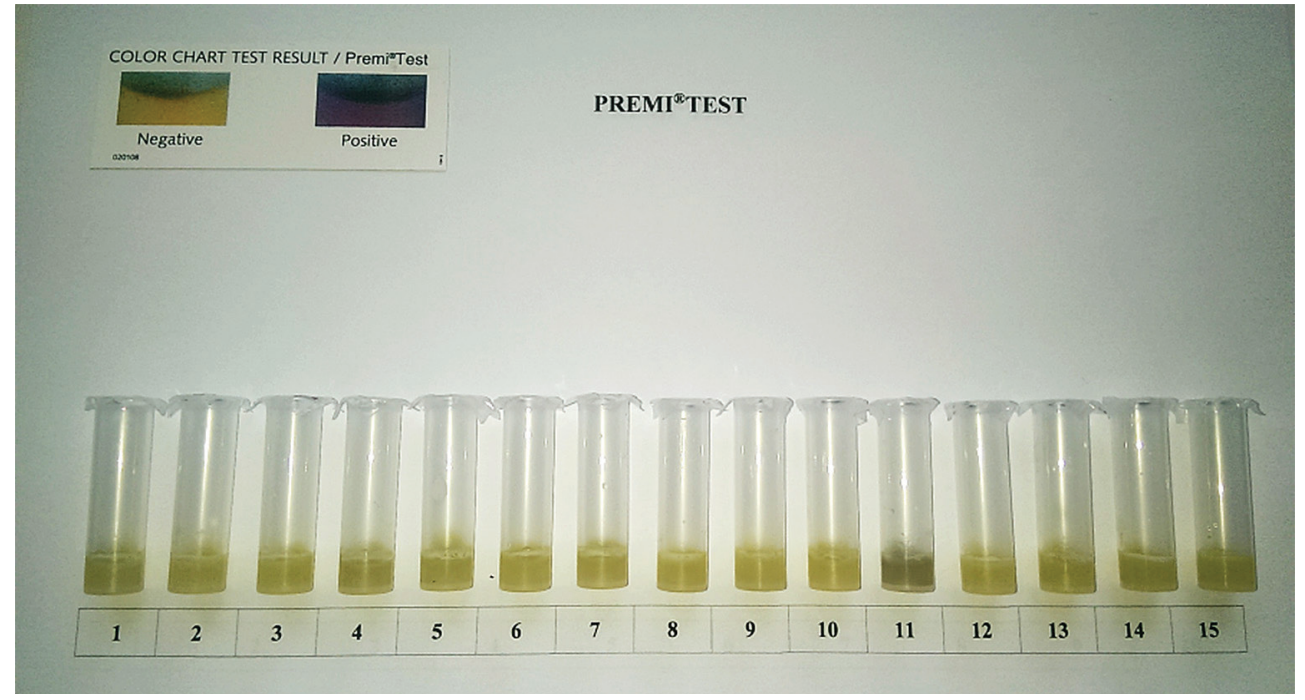

Fig. 2. Examination of raw cow milk for the presence/absence of antibiotic residues using the Premi ${ }^{\circledR}$ Test - Farm 1

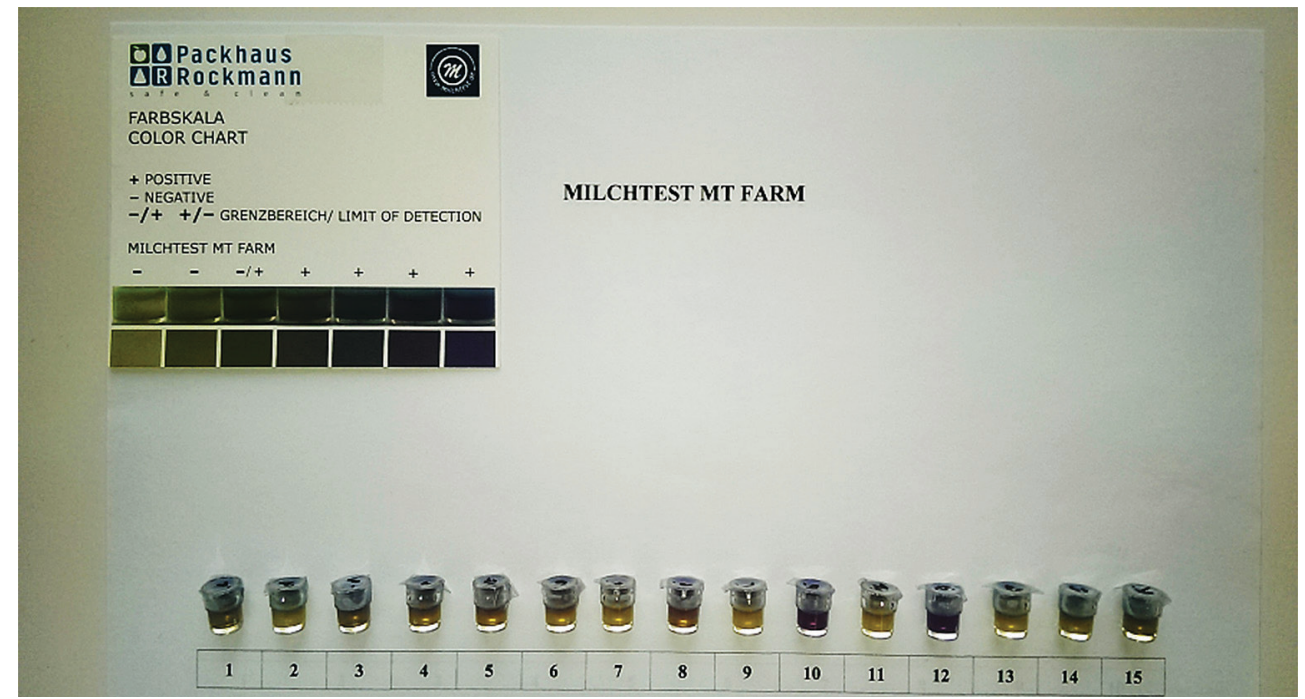

Fig. 3. Examination of raw cow's milk for the presence/absence of antibiotic residues using the Milchtest - Farm 2 


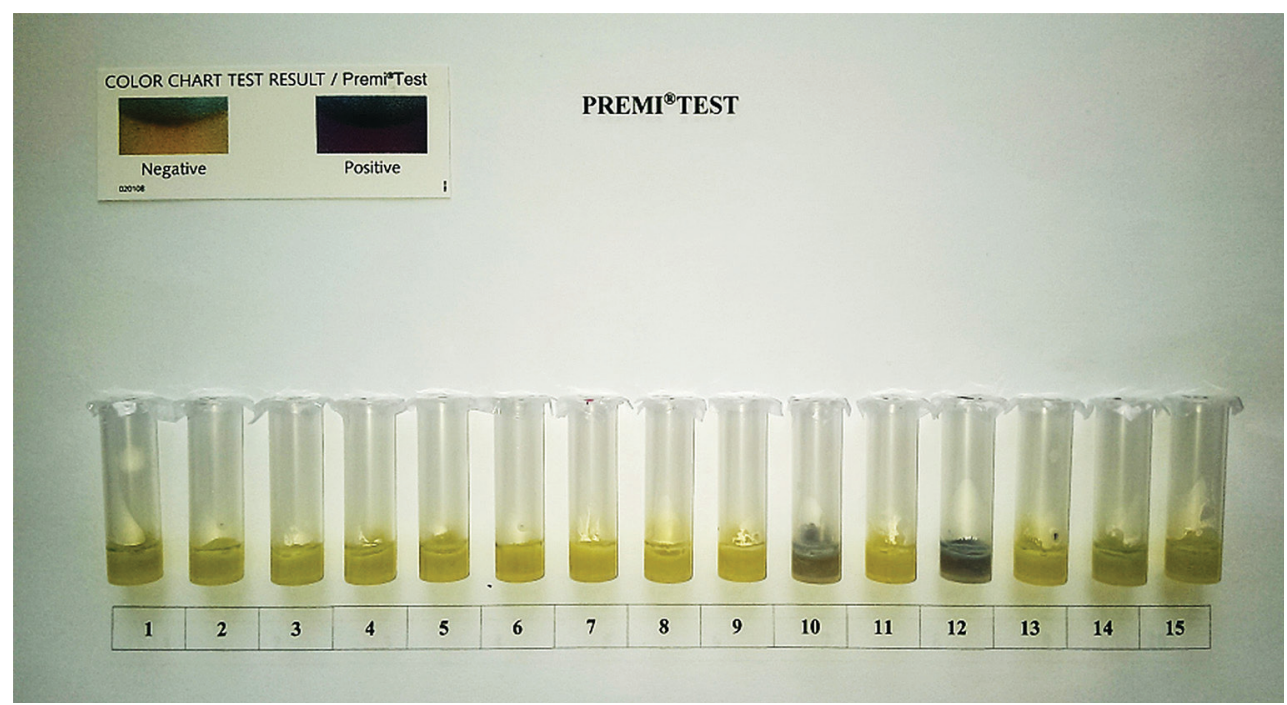

Fig. 4. Examination of raw cow's milk for the presence/absence of antibiotic residues using the Premi ${ }^{\circ}$ Test - Farm 2

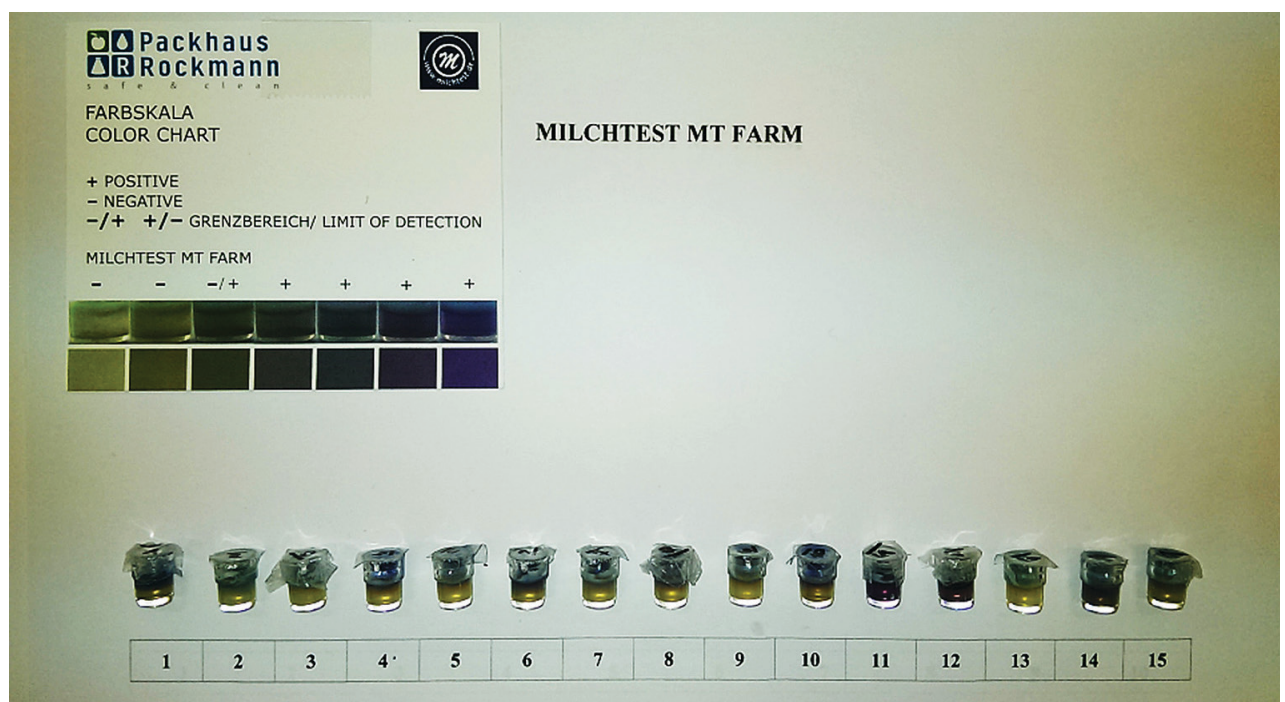

Fig. 5. Examination of raw cow's milk for the presence/absence of antibiotic residues using the Milchtest - Farm 3

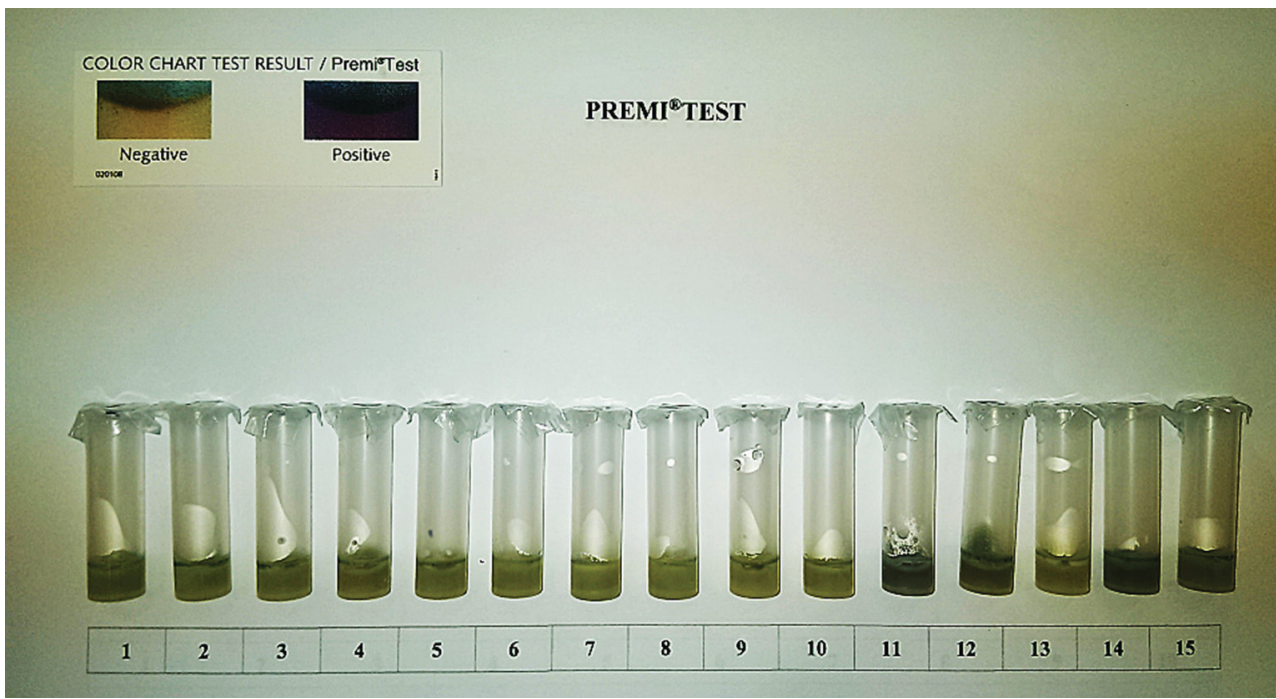

Fig. 6. Examination of raw cow's milk for the presence/absence of antibiotic residues using the Premi ${ }^{\circ}$ Test - Farm 3 


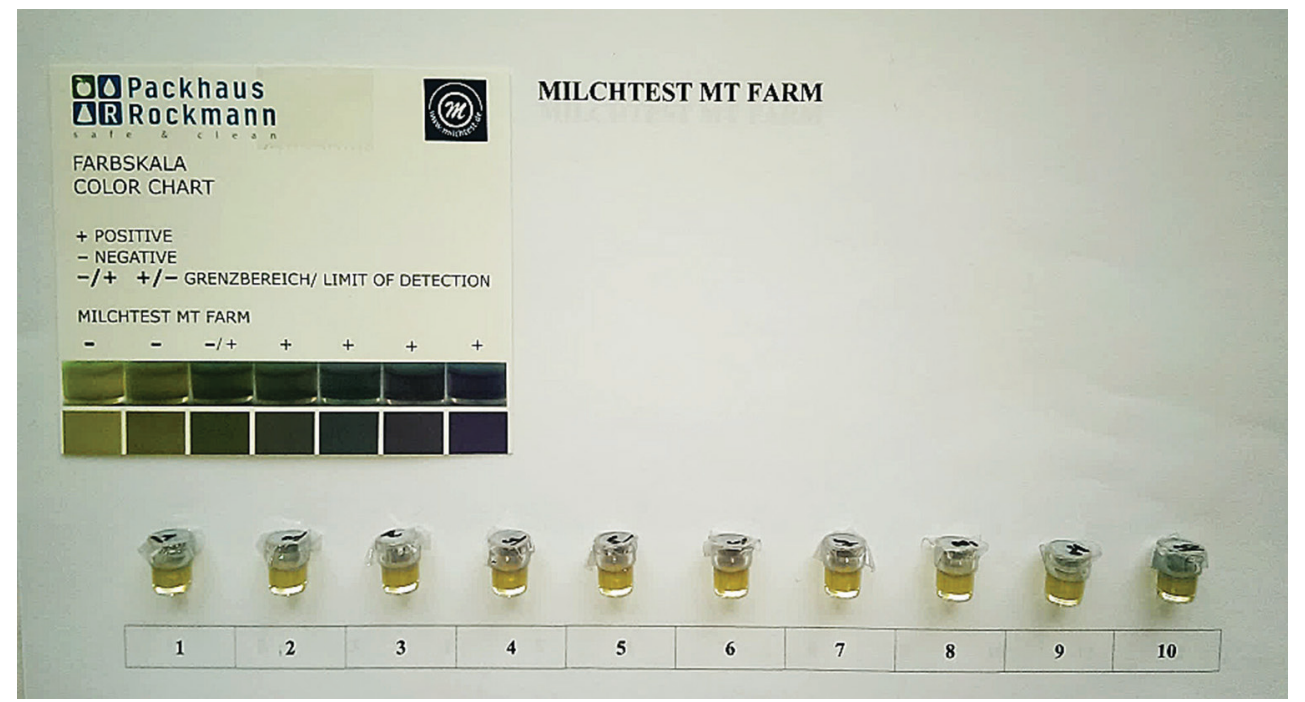

Fig. 7. Examination of cow's milk for the presence/absence of antibiotic residues using the Milchtest - grocery store

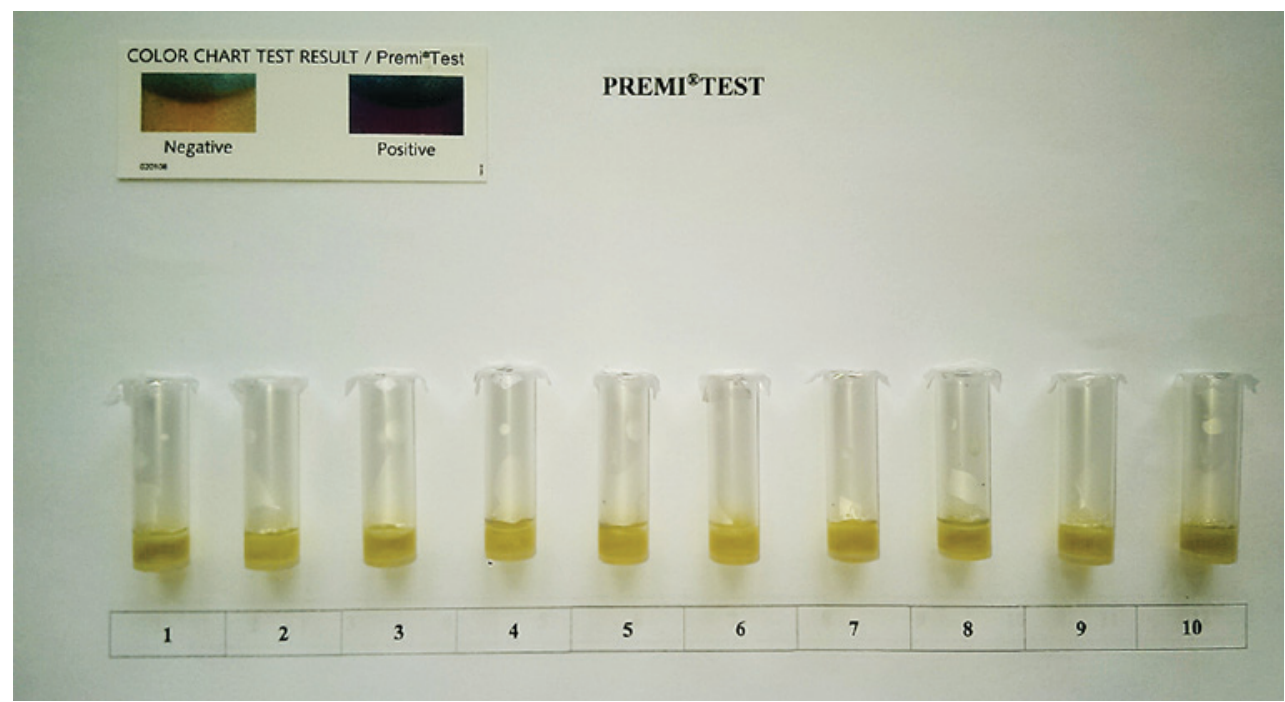

Fig. 8. Examination of cow's milk for the presence/absence of antibiotic residues using the Premi ${ }^{\circledR}$ Test - grocery store

milk and milk offered for sale. The test strain, Bacillus stearothermophilus var. calidolactis, used in these tests is generally considered the most susceptible microbial strain for broad-spectrum analysis of antibiotic residues. It showed the highest susceptibility particularly to those groups of antibiotics, for example beta-lactam antibiotics, that are most frequently used in veterinary practice to treat animals producing milk for human consumption $[2,6]$.

The rapid microbial inhibitory tests are a practical and valuable tool for the primary screening of antibiotic residues in milk, not only from the point of view of eliminating potential health risk, but also prevention of economic losses. They allow one within a short time to obtain results confirming or negating the presence of residue/residues of antibiotics in the milk samples. Although manufacturers of these rapid screening tests supply with them a relevant colour change scales declaring positive, dubious and negative results that support the objectivity of evaluation and the drawing of relevant conclusion, each positive or dubious result must be validated by confirmation analysis and result in adequate correction measures.

\section{CONCLUSIONS}

Our study compared the results of two commercial mi- 
crobial inhibitory tests, the Milchtest and Premi ${ }^{\oplus}$ Test, used for the detection of presence/absence of antibiotic residues in raw cow's milk and milk placed on the market. Both tests showed the presence of residues only in raw cow's milk obtained directly from agricultural farms. With regard to the higher number of positive and dubious results obtained with the Milchtest, this tests appeared more sensitive, however, if immediate screening and preliminary conclusions are needed, it is possible to use also the Premi ${ }^{\oplus}$ Test for the detection of the presence of antibiotics in milk intended for human consumption.

\section{ACKNOWLEDGEMENT}

The study was supported by the projects VEGA MŠVVaŠ SR and SAV No. 1/0576/17.

\section{REFERENCES}

1. Commission Regulation (EU) No. 37/2010 of 22 December 2009, on pharmacologically active substances and their classification regarding maximum residue limits in foodstuffs of animal origin. Official Journal of the European Union, L15, 2010, 1-72.

2. Gaudin, V., Maris, P., Fuselier, R., Ribouchon, J. L., Cadieu, N., Rault, A., 2004: Validation of microbiological method: the STAR protocol, a five-plate test, for the screening of antibiotic residues in milk. Food Addit. Contam., 21, 422-433.

3. http://www.milchtest.de/product/.
4. http://www.r-biopharm.com/products/food-feed-analysis/ residues/antibiotics/premitest/item/premitest-25.

5. Kardošová, A., 2010: Inspection of farm milk production hygiene (In Slovak). In Proceedings of lectures and posters of the international scientific conference Hygiena Alimentorum XXXI, May 25-27, University of Veterinary Medicine and Pharmacy, Košice, Slovakia, 275-277.

6. Pikkemaat, M. G., 2009: Microbial screening methods for detection of antibiotic residues in slaughter animals. Anal. Bioanal. Chem., 395, 893-905.

7. Poláková, Z., Kožárová, I., 2014: History of the development of screening methods (In Slovak). In Proceedings of lectures and posters of the XLIVth conference on food hygiene and technology "Lenfeldovy a Höklovy dny", October 15-16. Veterinary and Pharmaceutical University in Brno, Czechia, $150-154$.

8. Regulation (EC) No. 853/2004 of the European Parliament and of the Council of 29 April 2004 laying down specific hygiene rules for food of animal origin. Official Journal of the European Union, L139, 2004, 14-74.

9. Regulation (EC) No. 470/2009 of the European Parliament and of the Council of 6 May 2009 laying down Community procedures for the establishment of residue limits of pharmacologically active substances in foodstuffs of animal origin, repealing Council Regulation (EEC) No. 2377/90 and amending Directive 2001/82/EC of the European Parliament and of the Council and Regulation (EC) No. 726/2004 of the European Parliament and of the Council. Official Journal of the European Union, L152, 2009, 1-12.

10. R-26: Determination of residues of inhibitory substances in meat by Premi ${ }^{\oplus}$ Test method (In Slovak). http://www.svssr. sk/dokumenty/zakladne_info/Diagnostika/R\%2026\%20 PREMi\%20test.pdf.

Received May 22, 2017

Accepted August 19, 2017 